Article

\title{
A Novel Method for Determining the Attribute Weights in the Multiple Attribute Decision-Making with Neutrosophic Information through Maximizing the Generalized Single-Valued Neutrosophic Deviation
}

\author{
Wentao Xiong ${ }^{1, *}$ and Jing Cheng ${ }^{2}$ \\ 1 School of Mathematics and Statistics, Hubei Engineering University, Xiaogan 432000, China \\ 2 School of Economics and Management, Hubei Engineering University, Xiaogan 432000, China; \\ chengjing2003@126.com \\ * Correspondence: xiong2017@hbeu.edu.cn; Tel.:+86-712-2345-434
}

Received: 19 April 2018; Accepted: 1 June 2018; Published: 7 June 2018

\begin{abstract}
The purpose of this paper is to investigate the weights determination in the multiple attribute decision-making (MADM) with the single valued neutrosophic information. We first introduce a generalized single-valued neutrosophic deviation measure for a group of single valued neutrosophic sets (SVNSs), and then present a novel and simple nonlinear optimization model to determine the attribute weights by maximizing the total deviation of all attribute values, whether the attribute weights are partly known or completely unknown. Compared with the existing method based on the deviation measure, the presented approach does not normalize the optimal solution and is easier to integrate the subjective and objective information about attribute weights in the neutrosophic MADM problems. Moreover, the proposed nonlinear optimization model is solved to obtain an exact and straightforward formula for determining the attribute weights if the attribute weights are completely unknown. After the weights are obtained, the neutrosophic information of each alternative is aggregated by using the single valued neutrosophic weighted average (SVNWA) operator. In what follows, all alternatives are ranked and the most preferred one(s) is easily selected according to the score function and accuracy function. Finally, an example in literature is examined to verify the effectiveness and application of the developed approach. The example is also used to demonstrate the rationality for overcoming some drawbacks of the existing approach according to the maximizing deviation method.
\end{abstract}

Keywords: single valued neutrosophic set (SVNS); generalized single-valued neutrosophic deviation measure; multiple attribute decision-making; attribute weights

\section{Introduction}

Multiple attribute decision-making (MADM) or multiple criteria decision-making (MCDM) has been widely used for the purpose of ranking or selecting alternatives with respect to multiple and usually conflicting attributes (criteria). In order to deal with these problems, a lot of MADM methods have been presented and applied to many fields, including management science, decision sciences, economics, society, engineering, and so on. Classical MADM methods require the decision-maker (DM) to crisply evaluate the performance of alternatives under all attributes. However, the DM has to consider both quantitative and qualitative evaluations in several real problems. In addition, the information about the alternatives is often imprecise, inconsistent and subjective and the DM can only give approximate, incomplete information for some reasons. As a result, the imprecise and subjective data are usually presented and the alternatives are assessed in a fuzzy environment $[1,2]$. 
To deal with the imprecision and subjectivity inherent in the human decision-making process, several researchers suggested using the fuzzy set theory and intuitionistic fuzzy set theory for solving MADM problems. Bellman and Zadeh [3] first introduced the fuzzy set theory into multicriteria analysis to handle the imprecision and subjectivity of human decision-making. Since then, there have been tremendous efforts to develop the MADM models for the purpose of ranking all alternatives and selecting the preferred one(s). Many methodologies and their applications have been presented to various decision problems [4,5]. In addition, Atanassov and Rangasamy [6] extended the Zadeh fuzzy set and introduced the intuitionistic fuzzy set, which is suitable for addressing fuzziness and uncertainty in the MADM problems.

As the generalization of fuzzy set and intuitionistic fuzzy set, the neutrosophic set allows for handling "knowledge of neural thought" and can express incomplete, imprecise, and inconsistent information that was initially introduced by Smarandache [7]. Because it is difficult to directly apply the neutrosophic set in the real scientific and engineering problems, Wang et al. [8] defined a simplified form of neutrosophic set, called the single valued neutrosophic set (SVNS), and gave some various set theoretic operations and properties of SVNS. The SVNS is the subclasses of neutrosophic set for easy engineering applications. In order to get the more widely-used of neutrosophic algorithms, Broumi et al. [9] proposed Matlab toolboxes to compute the operational matrices in neutrosophic environments.

Recently, the SVNS has received more and more attention and been widely applied to the solution of the MADM problems [10]. Some crisp MADM methods were extended to handle the MADM problems with uncertain neutrosophic information, such as the technique for order preference by similarity to ideal solution (TOPSIS) method [11], the complex proportional assessment (COPRAS) approach [12], the maximizing deviation method [13], the grey relational analysis [14,15], the visekriterijumska optimizacija i kompromisno resenje (VIKOR) method [16], the outranking approach [17], etc. In addition, the different measures were defined and used to compare SVNSs in the MADM problem with neutrosophic information. For instance, Ye [18] developed the concept of correlation coefficient between SVNSs, and then applied the weighted correlation coefficient to solve the MCDM problem. Şahin and Liu [19] focused on the correlation and correlation coefficient of the single-valued neutrosophic hesitant fuzzy sets and investigated their basic properties. Aydogdu [20] introduced a similarity measure between two SVNSs and introduced an entropy concept of SVNSs. Jiang and Shou [21] presented the similarity measure between SVNS using the Dempster-Shafer evidence theory. Pramanik et al. [22] formulated a hybrid vector similarity measure by extending the concept of the variation coefficient similarity method [23] under both single valued neutrosophic and interval neutrosophic information. Most of the similarity measures and the weighted similarity measures were applied to solve the MADM/MCDM problems with SVNS information. Again, Ye [24] improved the cosine similarity measures of SNSs based on the cosine function, and applied them into the medical diagnosis problem. Şahin and Küçük [25] introduced a neurosophic subsethood measure for SVNSs based on the distance measure, and gave an application in the MCDM problem. Huang [26] defined a distance measure between two SVNSs by considering the truth membership function, indeterminacy-membership function, and falsity-membership function for the forward and backward differences. Şahin [27] defined the interval neutrosophic cross-entropy by extending the fuzzy and single valued neutrosophic cross-entropy, where the interval neutrosophic sets are converted into fuzzy sets and single valued neutrosophic sets, respectively. In addition, several aggregation operators and weighted aggregation operators [28-31] were presented to combine the different attribute values with the neutrosophic information and obtain the final ranking results of all alternatives.

In general, the weights of attributes are very important in the MADM methods, which can reflect the relative importance of attributes in the decision-making process and the different attribute weights can result in different ranking results. However, the above-mentioned MADM approaches provided the attribute weights directly by the DM. Usually, the weighting techniques can be divided into three categories: subjective methods, objective approaches and integrated methodologies [32]. The subjective 
methods determine attribute weights solely based on the decision-maker's expertise and preference information. Nevertheless, the objective approaches calculate the attribute weights according to the objective decision matrix information using some certain mathematical techniques. The integrated methodologies generate the attribute weights utilizing both the subjective preference information and the objective decision matrix information.

In literature, only a few methods deal with the weights of attributes in the MADM problems under neutrosophic environment. If the information about attribute weights is completely unknown, Küçük and Şahin [33] constructed an optimization model according to the traditional gray relational analysis (GRA) approach and generated an exact formula to calculate the attribute weights. Zhang et al. [34] utilized the entropy weight measure to obtain the objective weight and considered both the objective and subjective weight in the MADM problem under an interval-valued neutrosophic environment. Biswas et al. [35] established an optimization model to get the attribute weights based on the deviation between the alternatives and the ideal alternative in MADM problem, where the information about attribute weights is completely unknown. Ye [36] introduced two weighting models based on the distance-based similarity measures of SVNSs for deriving the weights of the decision makers and the attributes, where the decision matrices were represented by the form of SVNSs with completely unknown weight information. Zhang and Wu [11] established an optimization model using the maximizing deviation method, which can generate the optimal weights of criteria in their single valued neutrosophic TOPSIS method. Şahin and Liu [13] proposed two optimization models according to the maximizing deviation method to determine the attribute weights. If the attribute weights are not completely known, an optimal model was solved by the Lagrange method to provide a simple and exact equation. Different from the method in [13], this paper provides a new approach to determine the attribute weights based on the deviation of SVNSs in the decision-making problem with neutrosophic information. In this approach, a generalized single-valued neutrosophic deviation measure between two alternatives is introduced using the distance measure, and then an optimization model is established to generate the weights of attributes. Compared with the method in [13], the model is easier to combine the subjective information of attribute weights, and is more flexible to calculate the deviation of SVNSs with the different parameter $p$.

The remainder of this paper is organized as below. In the next section, we review some basic definitions and notations related to the SVNSs. In addition, we introduce the generalized single-valued neutrosophic deviation concept for a group of the SVNSs. In Section 3, we construct a new model to generate the objective attribute weights based on the generalized deviation measure of SVNSs, and apply the model to the MADM problem under neutrosophic environment, where the attribute values take the form of SVNSs and the information about attribute weights is partly known or completely unknown. In particular, the proposed optimization model is solved to obtain a simple and exact formula if the attribute weights are fully unknown. Moreover, in order to aggregate the neutrosophic information, the SVNWA operator is utilized to obtain the overall SVNSs for each alternative, and then the alternatives are further ranked according to the score function and accuracy function. In Section 4, a numerical example is used to illustrate the developed approach. It is also pointed out that the model in literature based on the deviation method may be infeasible in some cases. Finally, this paper is concluded in Section 5.

\section{Preliminaries}

In the section, we firstly review some basic concepts and notations related to the neutrosophic set and SVNSs, and then propose a generalized single-valued neutrosophic deviation measure between two alternatives, which will be used in the rest of this paper.

Definition 1 ([7]). Let $X$ be a finite universe of discourse, a neutrosophic set $N$ over $X$ is denoted by:

$$
N=\left\{<T_{N}(x), I_{N}(x), F_{N}(x)>\mid x \in X\right\},
$$


where the function $\left.T_{N}(x): X \rightarrow\right] 0^{-}, 1^{+}\left[, I_{N}(x): X \rightarrow\right] 0^{-}, 1^{+}\left[\right.$and $\left.F_{N}(x): X \rightarrow\right] 0^{-}, 1^{+}[$are truth-membership function, indeterminacy-membership function and falsity-membership function, respectively. The function values are real standard or non-standard subsets of $] 0^{-}, 1^{+}$, and satisfy $0^{-} \leq \sup T_{N}(x)+$ $\sup I_{N}(x)+\sup F_{N}(x) \leq 3^{+}$for each $x \in X$.

Since it is difficult to apply the neutrosophic set in some real decision-making problems, Wang et al. [8] presented the following concept of an SVNS, which is a subclass of the neutrosophic set according to Definition 1.

Definition 2 ([8]). Let X be a finite universe of discourse, an SVNS N over X is defined as below:

$$
N=\left\{<T_{N}(x), I_{N}(x), F_{N}(x)>\mid x \in X\right\},
$$

where the truth-membership function $T_{N}(x): X \rightarrow[0,1]$, the indeterminacy-membership function $I_{N}(x)$ : $X \rightarrow[0,1]$, and the falsity-membership function $F_{N}(x): X \rightarrow[0,1]$, satisfying the condition $0 \leq T_{N}(x)+$ $I_{N}(x)+F_{N}(x) \leq 3$ for each $x \in X$.

For convenience, the SVNS can be simplified as: $x=<T_{x}, I_{x}, F_{x}>$. In particular, the exact number 0 can be deemed the worst value, which is denoted as $(0,0,1)$ and the exact number 1 as the best value, denoted as $(1,0,0)$.

In addition, the following basic operations and relations are defined related to the SVNSs.

Definition 3 ([12]). Let $x=<T_{x}, I_{x}, F_{x}>$ and $y=<T_{y}, I_{y}, F_{y}>$ be any two SVNSs in $X$. Then the usual operations are defined as below:

(i) $x \oplus y=\left\langle T_{x}+T_{y}-T_{x} \cdot T_{y}, I_{x} \cdot I_{y}, F_{x} \cdot F_{y}\right\rangle$;

(ii) $x \otimes y=\left\langle T_{x} \cdot T_{y}, I_{x}+I_{y}-I_{x} \cdot I_{y}, F_{x}+F_{y}-F_{x} \cdot F_{y}\right\rangle$;

(iii) $\lambda \cdot x=\left\langle 1-\left(1-T_{x}\right)^{\lambda},\left(I_{x}\right)^{\lambda},\left(F_{x}\right)^{\lambda}\right\rangle, \lambda>0$;

(iv) $x^{\lambda}=\left\langle T_{x}^{\lambda}, 1-\left(1-I_{x}\right)^{\lambda}, 1-\left(1-F_{x}\right)^{\lambda}\right\rangle, \lambda>0$.

Definition 4 ([8]). Let $x=<T_{x}, I_{x}, F_{x}>$ and $y=<T_{y}, I_{y}, F_{y}>$ be any two SVNSs in $X . x \subseteq y$ if and only if $T_{x} \leq T_{y}, I_{x} \geq I_{y}$, and $F_{x} \geq F_{y}$ for every $x$ in $X$.

Moreover, Liu [28] presented the following aggregation operator, called single valued neutrosophic weighted average (SVNWA) operator.

Definition 5 ([28]). Let $x_{j}=<T_{x_{j}}, I_{x_{j}}, F_{x_{j}}>, j=1,2, \ldots, n$ be a collection of SVNSs. Then, the SVNWA operator is defined as a mapping SVNWA: $X^{n} \rightarrow X$, and the aggregated value using the SVNWA operator is expressed as below:

$$
\operatorname{SVNWA}_{\omega}\left(x_{1}, x_{2}, \ldots, x_{n}\right)=\left\langle 1-\prod_{j=1}^{n}\left(1-T_{x_{j}}\right)^{\omega_{j}}, I_{x_{j}}^{\omega_{j}}, F_{x_{j}}^{\omega_{j}}\right\rangle,
$$

where $\omega_{j}$ is the weight of $x_{j}, j=1,2, \ldots, n, \omega_{j} \in[0,1]$ and $\sum_{j=1}^{m} \omega_{j}=1$.

It is noted that the aggregation results of the SVNWA operator are still SVNSs. In particular, if $\omega_{j}=\frac{1}{n}, j=1,2, \ldots, n$, the SVNWA operator is called an arithmetic average operator.

In order to compare and rank the different SVNSs, a score function and an accuracy function were developed based on the truth-membership degree, indeterminacy-membership degree and falsity membership degree of SVNSs. The definition in $[37,38]$ is slightly modified and described as below: 
Definition 6. Let $x=<T_{x}, I_{x}, F_{x}>$ be an SVNS. Then, the score function $S(x)$ of $x$ is defined as below:

$$
S(x)=\left\{\begin{aligned}
\frac{2+T_{x}-I_{x}-F_{x}}{3}, & x \neq(0,0,0), \\
0, & x=(0,0,0),
\end{aligned}\right.
$$

where $S(x) \in[0,1]$, and $T_{x}, I_{x}$, and $F_{x}$ are the truth-membership degree, indeterminacy-membership degree and falsity membership degree, respectively.

Definition 7 ([37,38]). Let $x=<T_{x}, I_{x}, F_{x}>$ be an SVNS, the accuracy function $H(x)$ of $x$ is defined as below:

$$
H(x)=T_{x}-F_{x} \in[-1,1]
$$

where $T_{x}, I_{x}$, and $F_{x}$ is the truth-membership degree, indeterminacy-membership degree and falsity membership degree, respectively.

Generally, the larger the score $S(x)$, the greater the SVNS $x$, and the larger the value of $H(x)$, the more the accuracy degree of $x$. Based on the score function $S(x)$ and the accuracy function $H(x)$, the following definition is used to compare two SVNSs.

Definition 8 ([37,38]). Let $x=<T_{x}, I_{x}, F_{x}>$ and $y=<T_{y}, I_{y}, F_{y}>$ be two SVNSs. Assume that $S(x)$ and $S(y)$ are the score degrees of $x$ and $y$, and $H(x)$ and $H(y)$ are the accuracy degrees of $x$ and $y$, respectively. Then, we have:

(1) If $S(x)>S(y)$, then $x$ is superior to $y$, denoted by $x \succ y$;

(2) If $S(x)=S(y)$ and $H(x)=H(y)$, then $x$ is indifferent to $y$ with the same information, denoted by $x \simeq y$; If $S(x)=S(y)$ and $H(x)>H(y)$, then $x$ is greater than $y$, denoted by $x \succ y$.

\section{The Proposed Optimization Method for the Neutrosophic MADM Problem}

Suppose there are $n$ decision alternatives to be evaluated under $m$ different attributes in a MADM problem. Let $\mathcal{A}=\left\{A_{1}, A_{2}, \ldots, A_{n}\right\}$ be the set of alternatives and $\mathcal{G}=\left\{G_{1}, G_{2}, \ldots, G_{m}\right\}$ be the set of attributes. The evaluation information of decision alternatives in terms of the attributes can form a decision matrix denoted as $X=\left[x_{i j}\right]_{n \times m}=\left[<T_{x_{i j}}, I_{x_{i j}}, F_{x_{i j}}>\right]_{n \times m}$, where $x_{i j}=<T_{x_{i j}}, I_{x_{i j}}, F_{x_{i j}}>$ is the attribute value of $A_{i} \in \mathcal{A}$ with respect to $G_{j} \in \mathcal{G}$. The information is neutrosophic uncertain, and provided by the DM. $T_{x_{i j}}$ indicates the degree that the alternative $A_{i}$ should satisfy the attribute $G_{j} . F_{x_{i j}}$ indicates the degree that the alternative $A_{i}$ should not satisfy the attribute $G_{j}$, and $I_{x_{i j}}$ is neutrosophic uncertain degree of whether the alternative $A_{i}$ should satisfy the attribute $G_{j}$ or not. The purpose is to rank all alternatives and select the most preferred one(s). Let $\omega=\left\{\omega_{1}, \omega_{2}, \ldots, \omega_{m}\right\}$ be the attribute weights, $\omega_{j} \in[0,1]$ and $\sum_{j=1}^{m} \omega_{j}=1$. In this paper, we assume that all attributes are benefit attributes and the attribute weights are completely unknown or partly known. In what follows, this paper discusses the weight determination method by maximizing the total generalized deviation of all attribute values among the alternatives. The definition of generalized single-valued neutrosophic deviation measure between two alternatives is given based on the distance measure in [39]. 


\subsection{The Generalized Single-Valued Neutrosophic Deviation Measure between Two Alternatives}

Definition 9. Assume that $A_{s}$ and $A_{t}$ are any two alternatives under the attribute $G_{j} \in \mathcal{G}$, and the attribute values are the SVNSs, denoted by $x_{s j}=<T_{x_{s j}}(i), I_{x_{s j}}(i), F_{x_{s j}}(i)>$ and $x_{t j}=<T_{x_{t j}}(i), I_{x_{t j}}(i), F_{x_{t j}}(i)>$, $j=1,2, \ldots, m$, respectively. We have:

$$
d_{s t j}^{p}=\left\{\frac{1}{3} \sum_{i=1}^{n}\left(\left|T_{x_{s j}}(i)-T_{x_{t j}}(i)\right|^{p}+\left|I_{x_{s j}}(i)-I_{x_{t j}}(i)\right|^{p}+\left|F_{x_{s j}}(i)-F_{x_{t j}}(i)\right|^{p}\right)\right\}^{1 / p}
$$

where $p>0$ and $d_{s t j}^{p}$ is called as the generalized single-valued neutrosophic deviation measure.

If $p=1$, Equation (4) is reduced to the Hamming distance of two alternatives under the attribute $G_{j}$. If $p=2$, Equation (4) is reduced to the Euclidean distance of two alternatives under the attribute $G_{j}$. If $p=\infty$, Equation (4) can be written as the following formula:

$$
d_{s t j}^{p}=\max \left\{\left|T_{x_{s j}}(i)-T_{x_{t j}}(i)\right|,\left|I_{x_{s j}}(i)-I_{x_{t j}}(i)\right|,\left|F_{x_{s j}}(i)-F_{x_{t j}}(i)\right|\right\} .
$$

Thus, we have the following proposition for the generalized single-valued neutrosophic deviation measure:

(i) $0 \leq d_{s t j}^{p} \leq 1$;

(ii) $d_{s t j}^{p}=0$ if and only if $x_{s j}=x_{t j}$;

(ii) $d_{s t j}^{p}=d_{t s j}^{p}$;

(iv) If $x_{s j} \subseteq x_{t j} \subseteq x_{r j}, x_{r j}$ is an SVNS in $X$, then $d_{s r j}^{p} \geq d_{s t j}^{p}$ and $d_{s r j}^{p} \geq d_{t r j}^{p}$.

Proof. It is easy to verify that $d_{s t j}^{p}$ satisfies the properties $i>$-iii $>$. Therefore, we only prove $i v>$. Let $x_{s j} \subseteq x_{t j} \subseteq x_{r j}$. Then, $T_{x_{s j}}(i) \leq T_{x_{t j}}(i) \leq T_{x_{r j}}(i), I_{x_{s j}}(i) \geq I_{x_{t j}}(i) \geq I_{x_{r j}}(i)$, and $F_{x_{s j}}(i) \geq F_{x_{t j}}(i) \geq F_{x_{r j}}(i)$ for every $i \in X$ according to Definition 4 . Due to $p>0$, we have:

$$
\begin{gathered}
\left|T_{x_{s j}}(i)-T_{x_{t j}}(i)\right|^{p} \leq\left|T_{x_{s j}}(i)-T_{x_{r j}}(i)\right|^{p}, \quad\left|T_{x_{t j}}(i)-T_{x_{r j}}(i)\right|^{p} \leq\left|T_{x_{s j}}(i)-T_{x_{r j}}(i)\right|^{p}, \\
\left|I_{x_{s j}}(i)-I_{x_{t j}}(i)\right|^{p} \leq\left|I_{x_{s j}}(i)-I_{x_{r j}}(i)\right|^{p}, \quad\left|I_{x_{t j}}(i)-I_{x_{r j}}(i)\right|^{p} \leq\left|I_{x_{s j}}(i)-I_{x_{r j}}(i)\right|^{p}, \\
\left|F_{x_{s j}}(i)-F_{x_{t j}}(i)\right|^{p} \leq\left|F_{x_{s j}}(i)-F_{x_{r j}}(i)\right|^{p}, \quad\left|I_{x_{t j}}(i)-I_{x_{r j}}(i)\right|^{p} \leq\left|I_{x_{s j}}(i)-I_{x_{r j}}(i)\right|^{p},
\end{gathered}
$$

Thus, $\quad\left|T_{x_{s j}}(i)-T_{x_{r j}}(i)\right|^{p}+\left|I_{x_{s j}}(i)-I_{x_{r j}}(i)\right|^{p}+\left|F_{x_{s j}}(i)-F_{x_{r j}}(i)\right|^{p} \geq\left|T_{x_{s j}}(i)-T_{x_{t j}}(i)\right|^{p}+$ $\left|I_{x_{s j}}(i)-I_{x_{t j}}(i)\right|^{p}+\left|F_{x_{s j}}(i)-F_{x_{t j}}(i)\right|^{p}$, and $\left|T_{x_{s j}}(i)-T_{x_{r j}}(i)\right|^{p}+\left|I_{x_{s j}}(i)-I_{x_{r j}}(i)\right|^{p}+\left|I_{x_{s j}}(i)-I_{x_{r j}}(i)\right|^{p} \geq$ $\left|T_{x_{t j}}(i)-T_{x_{r j}}(i)\right|^{p}+\left|I_{x_{t j}}(i)-I_{x_{r j}}(i)\right|^{p}+\left|I_{x_{t j}}(i)-I_{x_{r j}}(i)\right|^{p}$.

Combining the above inequalities with Formula (4), we can get $d_{s r j}^{p} \geq d_{s t j}^{p}$ and $d_{s r j}^{p} \geq d_{t r j}^{p}$ for any $p>0$. That is, the property iv $>$ is satisfied. Hence, the proof is completed.

For all attributes in $\mathcal{G}$, a generalized single-valued neutrosophic deviation measure with the weight information is further given as below.

Definition 10. Assume that $A_{s}$ and $A_{t}$ are any two alternatives, $x_{s j}=<T_{x_{s j}}(i), I_{x_{s j}}(i), F_{x_{s j}}(i)>$ and $x_{t j}=<T_{x_{t j}}(i), I_{x_{t j}}(i), F_{x_{t j}}(i)>$ are the attribute values of $A_{s}$ and $A_{t}$ under the attribute $G_{j} \in \mathcal{G}$. A generalized single-valued neutrosophic deviation measure with weight information is defined as:

$$
\begin{aligned}
& d_{s t}^{p}=\sum_{j=1}^{m} \omega_{j}^{\alpha} d_{s t j}^{p} \\
& =\sum_{j=1}^{m} \omega_{j}^{\alpha}\left\{\frac{1}{3} \sum_{i=1}^{n}\left(\left|T_{x_{s j}}(i)-T_{x_{t j}}(i)\right|^{p}+\left|I_{x_{s j}}(i)-I_{x_{t j}}(i)\right|^{p}+\left|F_{x_{s j}}(i)-F_{x_{t j}}(i)\right|^{p}\right)\right\}^{1 / p},
\end{aligned}
$$


where $p>0$ and $\omega_{j}$ is the weight of the $j$ th attribute $G_{j} \in \mathcal{G} . \alpha$ belongs to $(0,1)$ for avoiding the extreme optimal solution in the following model (7). For convenience, let $\alpha=1 / 2$ in this paper.

\subsection{The Weight Determination by Maximizing the Generalized Deviation of Single Valued Neutrosophic Sets}

In several MCDM problems, the attribute weights play a very important role in ranking the alternatives. However, the weight information of attributes is completely unknown or partially available in some situations. When the weight information of attributes is partially available, they can usually be classified by the types in Table 1 , where $\alpha_{i}$ and $\varepsilon_{i}$ are nonnegative constants $\left\{0 \leq \alpha_{i} \leq \alpha_{i}+\varepsilon_{i} \leq 1\right\}$, and all the types are expressed by linear inequalities [13,40,41].

Table 1. The different types of attribute weights.

\begin{tabular}{|c|c|c|}
\hline & Ranking Relation & Form \\
\hline Form 1 & A weak ranking & $\left\{\omega_{i} \geq \omega_{j}\right\}$ \\
\hline Form 2 & A strict ranking & $\left\{\omega_{i}-\omega_{j} \geq \alpha_{i}\right\}$ \\
\hline Form 3 & A ranking of differences & $\left\{\omega_{i}-\omega_{j} \geq \omega_{k}-\omega_{l}\right\}$ for $j \neq k \neq l$ \\
\hline Form 4 & A ranking with multiples & $\left\{\omega_{i} \geq \alpha_{i} \omega_{j}\right\}$ \\
\hline Form 5 & An interval form & $\left\{\alpha_{i} \leq \omega_{i} \leq \alpha_{i}+\varepsilon_{i}\right\}$ \\
\hline
\end{tabular}

According to the above definition of generalized single-valued neutrosophic deviation, a maximizing deviation method is presented to discriminate the alternatives. That is, the bigger the total deviation of attribute values under the $j$ th attribute, the more the important degree of the $j$ th attibute and the larger the $j$ th attribute weight. Therefore, the following optimization model is constructed to maximize the sum $F(\omega)$ of the total deviation of the alternatives under all attributes:

$$
\begin{aligned}
& \max F(\omega)=\sum_{s=1}^{n} \sum_{t=1}^{n} d_{s t}^{p}=\sum_{s=1}^{n} \sum_{t=1}^{n} \sum_{j=1}^{n} \omega_{j}^{1 / 2} d_{s t j}^{p} \\
& \text { s.t. }\left\{\begin{array}{l}
\omega_{j} \in \Omega_{0} \\
\sum_{j=1}^{m} \omega_{j}=1 \\
0 \leq \omega_{j} \leq 1, j=1,2, \ldots, m,
\end{array}\right.
\end{aligned}
$$

where $p>0$, and $\Omega_{0}$ is the set of the information of attribute weights. The set of weight information is $\Omega_{0}=\varnothing$ if the information is completely unknown. When the weight information is partially available, $\Omega_{0} \neq \varnothing$. In this paper, we assume that they are expressed using the types in Table 1.

Let $\bar{\omega}_{j}=\omega_{j}^{1 / 2}, j=1,2, \ldots, m$. Model (7) is converted to the following model (8):

$$
\begin{array}{ll}
\max & F(\omega)=\sum_{s=1}^{n} \sum_{t=1}^{n} \sum_{j=1}^{n} \bar{\omega}_{j} d_{s t j}^{p} \\
\text { s.t. }\left\{\begin{array}{l}
\bar{\omega}_{j} \in \Omega \\
\sum_{j=1}^{m} \bar{\omega}_{j}^{2}=1 \\
0 \leq \bar{\omega}_{j} \leq 1, j=1,2, \ldots, m .
\end{array}\right.
\end{array}
$$

where $p>0$ and $\Omega$ is the corresponding set of weight information, where the weight values $\omega_{j}$ replaced by $\bar{\omega}_{j} \geq 0, j=1,2, \ldots, m$.

If $\Omega=\varnothing$ and $p=1$, model (7) can be rewritten as model (9). Model (9) is consistent with the model provided by [13], where $\bar{\omega}_{j}$ is the weight of the $j$ th attribute $G_{j}, j=1,2, \ldots, m$ in their 
model. However, the attribute weights do not satisfy the condition $\sum_{j=1}^{m} \bar{\omega}_{j}=1$, the obtained optimal solution of model (9) requires being normalized to calculate the attribute weights. On the other hand, the constraint condition $\sum_{j=1}^{m} \bar{\omega}_{j}^{2}=1$ can not be interpreted from the MADM viewpoint. Thus, the subjective weight information can not be added directly in model (9):

$$
\begin{array}{ll}
\max & F(\omega)=\sum_{s=1}^{n} \sum_{t=1}^{n} \sum_{j=1}^{n} \bar{\omega}_{j} d_{s t j^{p}}^{p} \\
\text { s.t. }\left\{\begin{array}{l}
\sum_{j=1}^{m} \bar{\omega}_{j}^{2}=1, \\
0 \leq \bar{\omega}_{j} \leq 1, j=1,2, \ldots, m .
\end{array}\right.
\end{array}
$$

In order to solve model (9), the following Lagrange function is easily constructed:

$$
L(\omega, \lambda)=\sum_{s=1}^{n} \sum_{t=1}^{n} \sum_{j=1}^{n} \bar{\omega}_{j} d_{s t j}^{p}+\frac{\lambda}{2}\left(\sum_{j=1}^{m} \bar{\omega}_{j}^{2}-1\right)
$$

where $\lambda$ is the Lagrange multiplier.

In what follows, the partial derivatives of $L(\omega, \lambda)$ are calculated as below:

$$
\left\{\begin{array}{l}
\frac{\partial L}{\partial \bar{\omega}_{j}}=\sum_{s=1}^{n} \sum_{t=1}^{n} d_{s t j}^{p}+\lambda \bar{\omega}_{j}=0 \\
\frac{\partial L}{\partial \lambda}=\sum_{j=1}^{m} \bar{\omega}_{j}^{2}-1=0 .
\end{array}\right.
$$

From Equation (11), the following simple and exact formula is obtained for determining the attribute weights:

$$
\bar{\omega}_{j}^{*}=\frac{\sum_{s=1}^{n} \sum_{t=1}^{n} d_{s t j}^{p}}{\sqrt{\sum_{j=1}^{m}\left(\sum_{s=1}^{n} \sum_{t=1}^{n} d_{s t j}^{p}\right)^{2}}}, j=1,2, \ldots, m .
$$

Furthermore, the attribute weights are determined as:

$$
\begin{aligned}
& \omega_{j}=\left(\bar{\omega}_{j}^{*}\right)^{2}=\frac{\left(\sum_{s=1}^{n} \sum_{t=1}^{n} d_{s t j}^{p}\right)^{2}}{\sum_{j=1}^{m}\left(\sum_{s=1}^{n} \sum_{t=1}^{n} d_{s t j}^{p}\right)^{2}} \\
& =\frac{\left(\sum_{s=1}^{n} \sum_{t=1}^{n}\left(\sum_{i=1}^{n}\left(\left|T_{x_{s j}}(i)-T_{x_{t j}}(i)\right|^{p}+\left|I_{x_{s j}}(i)-I_{x_{t j}}(i)\right|^{p}+\left|F_{x_{s j}}(i)-F_{x_{t j}}(i)\right|^{p}\right)\right)^{1 / p}\right)^{2}}{\sum_{j=1}^{m}\left(\sum_{s=1}^{n} \sum_{t=1}^{n}\left(\sum_{i=1}^{n}\left(\left|T_{x_{s j}}(i)-T_{x_{t j}}(i)\right|^{p}+\left|I_{x_{s j}}(i)-I_{x_{t j}}(i)\right|^{p}+\left|F_{x_{s j}}(i)-F_{x_{t j}}(i)\right|^{p}\right)\right)^{1 / p}\right)^{2}}, j=1,2, \ldots, m .
\end{aligned}
$$

If $\Omega_{0} \neq \varnothing$, the constraints set $\Omega$ is also nonempty. Thus, model (7) is nonlinear, but it is easy to be solved using the optimization softwares such as LINGO and MATLAB. After the optimal solution is generated, the weights of all attributes are further calculated using the formula $\omega_{j}=\left(\bar{\omega}_{j}^{*}\right)^{2}$, $j=1,2, \ldots, m$.

\subsection{The Method for Solving the Neutrosophic MADM Problems}

In a real situation, a MADM problem is formed based on the main steps [42], including: (i) establish the set of evaluation attributes $\mathcal{G}$; (ii) develop the set of alternatives $\mathcal{A}$; (iii) obtain performance data for evaluation alternatives in terms of all attributes. We assume that the data denotes by SVNSs and forms a neutrosophic decision matrix $X=\left[x_{i j}\right]_{n \times m}=\left[<T_{x_{i j}}, I_{x_{i j}}, F_{x_{i j}}>\right]_{n \times m}$, where $x_{i j}$ is the attribute value for the alternative $A_{i} \in \mathcal{A}$ with respect to the attribute $G_{j} \in \mathcal{G}$; 
(iv) develop the subjective information about attribute weights, which is completely unknown or partly known. If the weight information is partly known, let $\Omega_{0}$ is the set of weight information, constructed by the forms 1-5 in Table 1; and (v) apply some certain MADM method. In this subsection, we discuss step (v) and develop a practical method to solve the MADM problem. A procedure for ranking all alternatives and choosing the best preferred one(s) can be described as below:

Step 1. Determine the set of weights $\Omega$ according to the available weight information. If the information about the attribute weights is fully unknown, let $\Omega=\varnothing$, and go to Step 2 . Otherwise, the set $\Omega_{0}$ is converted to the set $\Omega$ if the information of the attribute weights is partly known, go to Step 3.

Step 2. Give the parameter $p$ and calculate the generalized single-valued neutrosophic deviation measure of all attribute values using Equation (13).

Step 3. Give the parameter $p$ and solve model (8) to obtain the optimal solution $\bar{\omega}_{j}^{*}, j=1,2, \ldots, m$, and then the attribute weights are computed using the formular $\omega_{j}=\left(\bar{\omega}_{j}^{*}\right)^{2}, j=1,2, \ldots, m$.

Step 4. Compute the overall evaluation value $r_{i}$ of the alternative $A_{i}, i=1,2, \ldots, n$ using the obtained the attribute weights $\omega_{j}, j=1,2, \ldots, m$ according to the SVNWA operator, expressed by Equation (1).

Step 5. Calculate the scores degree $S_{i}$ and the accuracy degree $H_{i}$ of the overall evaluation value $r_{i}$.

Step 6. Rank all alternatives $A_{i}, i=1,2, \ldots, n$, and then select the most preferred one(s) using the scores and accuracy degrees according to Definition 8.

Step 7. End.

\section{An Illustrative Example}

In this section, an example is used to illustrate the proposed method, where three cases are considered to rank all alternatives and select the preferred one(s). In Case 1, suppose that the weights of attributes are completely unknown, the exact Equation (13) can be used to calculate the weights. In Case 2 and Case 3, we assume that the weight information is partly available and the attribute weights are derived to rank all alternatives. It is also pointed out that the model in literature is invalid in Case 3, where most weights of attributes in their model are 0 , and only an attribute weight is 1.

Consider the MADM problem adapted from Şahin and Liu [13], where four suppliers are evaluated for an automotive company to select the most appropriate supplier for one of the key elements in its manufacturing process. In order to assess these alternative suppliers, four attributes are considered, such as: (1) product quality $\left(G_{1}\right),(2)$ relationship closeness $\left(G_{2}\right)$, (3) delivery performance $\left(G_{3}\right)$, and (4) price $\left(G_{4}\right)$. Şahin and Liu [13] assumed that four decision makers use the SVNSs to evaluate the four possible alternatives $A_{i},(i=1,2,3,4)$ and the ranking was obtained by the aggregated overall SVNSs using the SVNWA operator. Here, we suppose that the decision matrices of four decision makers are first aggregated using SVNWA operator based on their weight vector $\lambda=\left(\frac{1}{4}, \frac{1}{4}, \frac{1}{4}, \frac{1}{4}\right)^{T}$. The aggregated decision matrix is listed in Table 2, where the data is rounded to two decimal places. Based on the proposed approach, the following steps are given to rank all alternatives and obtain the most desirable or preferred alternative(s).

Table 2. The aggregated decision matrix given by four decision makers.

\begin{tabular}{ccccc}
\hline & $G_{1}$ & $G_{2}$ & $G_{3}$ & $G_{4}$ \\
\hline$A_{1}$ & $(0.26,0.22,0.26)$ & $(0.38,0.14,0.34)$ & $(0.20,0.22,0.52)$ & $(0.46,0.19,0.42)$ \\
$A_{2}$ & $(0.47,0.15,0.28)$ & $(0.54,0.26,0.28)$ & $(0.35,0.26,0.16)$ & $(0.44,0.16,0.30)$ \\
$A_{3}$ & $(0.360 .28,0.32)$ & $(0.34,0.24,0.32)$ & $(0.35,0.33,0.28)$ & $(0.27,0.42,0.23)$ \\
$A_{4}$ & $(0.41,0.26,0.12)$ & $(0.38,0.130 .26)$ & $(0.26,0.34,0.31)$ & $(0.40,0.26,0.19)$ \\
\hline
\end{tabular}

Case 1: If the attribute weights are completely unknown, we use the proposed method to rank all alternatives and determine the most preferred alternative(s). 
Step 1. Because the attribute weights are entirely unknown, the set of weight information is empty. That is, $\Omega_{0}=\Omega=\varnothing$.

Step 2. Give the value of $p$, and calculate the generalized single-valued neutrosophic deviation value $d_{s t j}^{p}$ of all alternatives under each attribute using Equation (4).

Step 3. Utilize Equation (13) to obtain the vector of attribute weights:

If $p=1$, we have $\omega=(0.2119,0.1329,0.3063,0.3489)^{T}$. If $p=2$, we have $\omega=$ $(0.2169,0.1463,0.3098,0.3270)^{T}$, and, if $p=\infty$, we have $\omega=(0.2170,0.1577,0.3291,0.2962)^{T}$.

Step 4. Compute the overall SVNS $r_{i}$ of all alternatives $A_{i}, i=1,2,3,4$ according to the above weight vector and the SVNWA operator using Equation (1). The results are shown in the second, the fourth and the sixth columns of Table 3.

Step 5. Calculate the scores of the collective overall SVNSs $r_{i}$ of all alternatives by the SVNWA operator based on Definition 5, respectively. The results are presented in the third, the fifth and the seventh columns of Table 3.

Step 6. Rank all the alternatives in accordance with the scores the overall SVNSs $r_{i}, i=1,2,3,4$. As can be seen in Table 3, the ranking result is the same calculated by the SVNWA operator with the different parameter $p$. The ranking is also consistent with the ranking of the results in [13]. Therefore, we have: $A_{2} \succ A_{4} \succ A_{1} \succ A_{3}$ and the most preferred alternative is $A_{2}$.

Table 3. The overall SVNSs and associated rankings (in parentheses) with respect to completely unknown attribute weights.

\begin{tabular}{|c|c|c|c|c|c|c|c|}
\hline & The Overall SVNSs & Scores & The Overall SVNSs & Scores & The Overall SVNSs & Scores & Scores in [13] \\
\hline & $p=1$ & & $p=2$ & & $p=\infty$ & & \\
\hline$r_{1}$ & $(0.3368,0.1968,0.3939)$ & $0.5820(3)$ & $(0.3336,0.1963,0.3921)$ & $0.5817(3)$ & $(0.3275,0.1962,0.3927)$ & $0.5795(3)$ & $0.2895(3)$ \\
\hline$r_{2}$ & $(0.4356,0.1953,0.2416)$ & $0.6662(1)$ & $(0.4369,0.1969,0.2408)$ & $0.6664(1)$ & $(0.4366,0.1998,0.2377)$ & $0.6663(1)$ & $0.4075(1)$ \\
\hline$r_{3}$ & $(0.3240,0.3323,0.2737)$ & $0.5726(4)$ & $(0.3256,0.3289,0.2756)$ & $0.5737(4)$ & $(0.3279,0.3253,0.2777)$ & $0.5750(4)$ & $0.1881(4)$ \\
\hline$r_{4}$ & $(0.3597,0.2574,0.2088)$ & $0.6312(2)$ & $(0.3590,0.2553,0.2095)$ & $0.6314(2)$ & $(0.3561,0.2546,0.2123)$ & $0.6298(2)$ & $0.3345(2)$ \\
\hline
\end{tabular}

Case 2: Assume that the attribute weights are partly known and the set $\Omega_{0}$ of all known weight information is given as below:

Step 1. Let $\Omega=\left\{\begin{array}{l}\bar{\omega}_{1}^{2} \in[0.18,0.20], \bar{\omega}_{2}^{2} \in[0.15,0.25], \bar{\omega}_{3}^{2} \in[0.30,0.35], \\ \bar{\omega}_{4}^{2} \in[0.30,0.40], \sum_{i=1}^{4} \bar{\omega}_{i}^{2}=1 .\end{array}\right\}$

Step 2. The results of the generalized single-valued neutrosophic deviation measure of all alternatives under each attribute are the same as that calculated in Step 2 in Case 1. Namely, if $p=1$, we have $\sum_{s=1}^{n} \sum_{t=s+1}^{n} d_{s t 1}^{1}=0.5767, \sum_{s=1}^{n} \sum_{t=s+1}^{n} d_{s t 2}^{1}=0.4567, \sum_{s=1}^{n} \sum_{t=s+1}^{n} d_{s t 3}^{1}=0.6933, \sum_{s=1}^{n} \sum_{t=s+1}^{n} d_{s t 4}^{1}=0.74$. If $p=2$, we have $\sum_{s=1}^{n} \sum_{t=s+1}^{n} d_{s t 1}^{2}=0.3826, \sum_{s=1}^{n} \sum_{t=s+1}^{n} d_{s t 2}^{2}=0.3142, \sum_{s=1}^{n} \sum_{t=s+1}^{n} d_{s t 3}^{2}=0.4572$, $\sum_{s=1}^{n} \sum_{t=s+1}^{n} d_{s t 4}^{2}=0.4697$. If $p=\infty$, we have $\sum_{s=1}^{n} \sum_{t=s+1}^{n} d_{s t 1}^{3}=0.95, \sum_{s=1}^{n} \sum_{t=s+1}^{n} d_{s t 2}^{3}=0.81$, $\sum_{s=1}^{n} \sum_{t=s+1}^{n} d_{s t 3}^{3}=1.17, \sum_{s=1}^{n} \sum_{t=s+1}^{n} d_{s t 4}^{3}=1.11$.

Step 3. Utilize the model (8) to establish the following nonlinear programming model with the different parameter $p$. For instance, the following model (14) is constructed if $p=1$ :

$$
\begin{array}{ll}
\max & d(\omega)=0.5767 \bar{\omega}_{1}+0.4567 \bar{\omega}_{2}+0.6933 \bar{\omega}_{3}+0.74 \bar{\omega}_{4} \\
\text { s.t. }\left\{\begin{array}{l}
0.18 \leq \bar{\omega}_{1}^{2} \leq 0.20,0.15 \leq \bar{\omega}_{2}^{2} \leq 0.25 \\
0.30 \leq \bar{\omega}_{3}^{2} \leq 0.35,0.30 \leq \bar{\omega}_{4}^{2} \leq 0.40 \\
\sum_{j=1}^{4} \bar{\omega}_{j}^{2}=1,0 \leq \bar{\omega}_{j} \leq 1, j=1,2,3,4
\end{array}\right.
\end{array}
$$


Solving this above model (14), we obtain the optimal solution $\bar{\omega}^{*}=(0.4472,0.3873,0.5512,0.5884)^{T}$. Thus, the attribute weights are calculated as: $\omega_{1}=0.20, \omega_{2}=0.15, \omega_{3}=0.3038, \omega_{4}=0.3462$, respectively, based on the formula $\omega_{j}=\left(\bar{\omega}_{j}^{*}\right)^{2}, j=1,2, \ldots, m$. Similarly, we can obtain the attribute weights $\omega_{1}=0.20, \omega_{2}=0.15, \omega_{3}=0.3162, \omega_{4}=0.3338$ for $p=2$ and $\omega_{1}=0.20, \omega_{2}=0.1611$, $\omega_{3}=0.3362, \omega_{4}=0.3026$ for $p=\infty$.

Step 4. Calculate the overall SVNS $r_{i}$ of all the alternatives $A_{i}, i=1,2,3,4$ according to the attribute weights and the SVNWA operator using Equation (1). The results are shown in the second, the fourth and the sixth columns of Table 4 with the different parameter $p$.

Step 5. Calculate the scores of the collective overall SVNSs $r_{i}$ of all alternatives by the SVNWA operator based on Definition 5, respectively. The results are shown in Table 4.

Step 6. Rank all the alternatives in accordance with the scores the overall SVNSs $r_{i}, i=1,2,3,4$. As shown in Table 4, the ranking result is the same as that obtained by the SVNWA operator, despite their scores are different with the different parameter $p$. The ranking is also consistent with the ranking of the result in [13]. Therefore, we have: $A_{2} \succ A_{4} \succ A_{1} \succ A_{3}$ and the most preferred alternative is $A_{2}$.

Table 4. The overall SVNSs and associated rankings (in parentheses) with respect to partly known attribute weights.

\begin{tabular}{cccccccc}
\hline \multicolumn{2}{c}{ Inputs } & \multicolumn{5}{c}{ Outputs } \\
\hline & The Overall SVNSs & Scores & The Overall SVNSs & Scores & The Overall SVNSs & Scores & Scores in [13] \\
\hline \multicolumn{2}{c}{$\boldsymbol{p}=\mathbf{1}$} & & $\boldsymbol{p}=\mathbf{2}$ & & $\boldsymbol{p}=\boldsymbol{}$ \\
\hline$r_{1}$ & $(0.3384,0.1954,0.3945)$ & $0.5828(3)$ & $(0.3351,0.1958,0.3955)$ & $0.5813(3)$ & $(0.3288,0.1957,0.3963)$ & $0.5790(3)$ & $0.2848(3)$ \\
$r_{2}$ & $(0.4373,0.1969,0.2419)$ & $0.6662(1)$ & $(0.4363,0.1981,0.2400)$ & $0.6661(1)$ & $(0.4358,0.2011,0.2369)$ & $0.6660(1)$ & $0.4074(1)$ \\
$r_{3}$ & $(0.3239,0.3309,0.2741)$ & $0.5730(4)$ & $(0.3249,0.3300,0.2748)$ & $0.5734(4)$ & $(0.3272,0.3263,0.2769)$ & $0.5747(4)$ & $0.1798(4)$ \\
$r_{4}$ & $(0.3595,0.2542,0.2108)$ & $0.6315(2)$ & $(0.3579,0.2551,0.2121)$ & $0.6302(2)$ & $(0.3549,0.2545,0.2149)$ & $0.6285(2)$ & $0.3265(2)$ \\
\hline
\end{tabular}

Case 3: Assume that the attribute weights are partly known and the set $\Omega_{0}$ of all known weight information is given as follows:

Step 1. Let $\Omega=\left\{\begin{array}{l}\bar{\omega}_{1}^{2} \leq 2 \bar{\omega}_{2}^{2}, \bar{\omega}_{1}^{2}+\bar{\omega}_{2}^{2} \leq \bar{\omega}_{3}^{2}, \\ \bar{\omega}_{3}^{2}-\bar{\omega}_{1}^{2} \geq 0.2, \sum_{i=1}^{4} \bar{\omega}_{i}^{2}=1 .\end{array}\right\}$

Step 2. The results of the generalized single-valued neutrosophic deviation measure of all alternatives under each attribute are the same as that calculated in Step 2 in Case 1. That is, if $p=1$, we have $\sum_{s=1}^{n} \sum_{t=s+1}^{n} d_{s t 1}^{1}=0.5767, \sum_{s=1}^{n} \sum_{t=s+1}^{n} d_{s t 2}^{1}=0.4567, \sum_{s=1}^{n} \sum_{t=s+1}^{n} d_{s t 3}^{1}=0.6933$, $\sum_{s=1}^{n} \sum_{t=s+1}^{n} d_{s t 4}^{1}=0.74$. If $p=2$, we have $\sum_{s=1}^{n} \sum_{t=s+1}^{n} d_{s t 1}^{2}=0.3826, \sum_{s=1}^{n} \sum_{t=s+1}^{n} d_{s t 2}^{2}=0.3142$, $\sum_{s=1}^{n} \sum_{t=s+1}^{n} d_{s t 3}^{2}=0.4572, \sum_{s=1}^{n} \sum_{t=s+1}^{n} d_{s t 4}^{2}=0.4697$. If $p=\infty$, we have $\sum_{s=1}^{n} \sum_{t=s+1}^{n} d_{s t 1}^{3}=0.95$, $\sum_{s=1}^{n} \sum_{t=s+1}^{n} d_{s t 2}^{3}=0.81, \sum_{s=1}^{n} \sum_{t=s+1}^{n} d_{s t 3}^{3}=1.17, \sum_{s=1}^{n} \sum_{t=s+1}^{n} d_{s t 4}^{3}=1.11$

Step 3. Utilize model (8) to formulate the following nonlinear programming model:

$$
\begin{array}{ll}
\max & d(\omega)=0.5767 \bar{\omega}_{1}+0.4567 \bar{\omega}_{2}+0.6933 \bar{\omega}_{3}+0.74 \bar{\omega}_{4}, \\
\text { s.t. }\left\{\begin{array}{l}
\bar{\omega}_{1}^{2} \leq 2 \bar{\omega}_{2}^{2}, \bar{\omega}_{1}^{2}+\bar{\omega}_{2}^{2} \leq \bar{\omega}_{3}^{2}, \\
\bar{\omega}_{3}^{2}-\bar{\omega}_{1}^{2} \geq 0.2, \\
\sum_{j=1}^{4} \bar{\omega}_{j}^{2}=1,0 \leq \bar{\omega}_{j} \leq 1, j=1,2,3,4 .
\end{array}\right.
\end{array}
$$


Solving this model (15), we obtain the optimal solution $\bar{\omega}^{*}=(0.4243,0.3873,0.5871,0.5703)^{T}$. However, the optimal solution is calculated by the model in [13] is $\omega^{*}=(0,0,1,0)^{T}$. This means that only the performance values under the attribute $G_{3}$ are used to evaluate these alternatives, whereas the attributes values under the other attributes are neglected. Obviously, it is infeasible. Using the presented model (8), if $p=1$, the attribute weights are computed as: $\omega_{1}=0.0736$, $\omega_{2}=0.2736, \omega_{3}=0.3471, \omega_{4}=0.3058$ based on the formula $\omega_{j}=\left(\bar{\omega}_{j}^{*}\right)^{2}, j=1,2, \ldots, m$. Similarly, we can obtain the attribute weights $\omega_{1}=0.0779, \omega_{2}=0.2779, \omega_{3}=0.3559$, $\omega_{4}=0.2883$ for $p=2$ and $\omega_{1}=0.0841, \omega_{2}=0.2841, \omega_{3}=0.3683, \omega_{4}=0.2643$ for $p=\infty$.

Step 4. According to the above attribute weights and the SVNWA operator Equation (1), we obtain the overall SVNS $r_{i}$ of all the alternatives $A_{i}, i=1,2,3,4$, as shown in Table 5.

Step 5. Calculate the score degrees of the collective overall SVNSs $r_{i}$ of all alternatives by the SVNWA operator based on Definition 5, respectively. The results are shown in the third, the fifth and the seventh columns of Table 5 .

Step 6. Rank all the alternatives in accordance with the score degrees of the overall SVNSs $r_{i}, i=1,2,3,4$. As shown in Table 3, the ranking result is the same as that obtained by the SVNWA operator with the different parameter $p$, despite their scores are different. Therefore, we have: $A_{2} \succ A_{4} \succ A_{1} \succ A_{3}$ and the most preferred alternative is $A_{2}$.

Table 5. The overall SVNSs and associated rankings (in parentheses) with respect to partly known attribute weights.

\begin{tabular}{ccccccc}
\hline & The Overall SVNSs & Scores & The Overall SVNSs & Scores & The Overall SVNSs & Scores \\
\hline \multicolumn{2}{c}{$\boldsymbol{p}=\mathbf{1}$} & & $\boldsymbol{p}=\mathbf{2}$ & $\boldsymbol{p}=\boldsymbol{}$ \\
\hline$r_{1}$ & $(0.3422,0.1859,0.4121)$ & $0.5814(3)$ & $(0.3386,0.1860,0.4116)$ & $0.5803(3)$ & $(0.3335,0.1862,0.4110)$ & $0.5788(3)$ \\
$r_{2}$ & $(0.4434,0.2152,0.2355)$ & $0.6642(1)$ & $(0.4433,0.2166,0.2340)$ & $0.6642(1)$ & $(0.4431,0.2184,0.2320)$ & $0.6642(1)$ \\
$r_{3}$ & $(0.3245,0.3217,0.2762)$ & $0.5755(4)$ & $(0.3258,0.3197,0.2774)$ & $0.5762(4)$ & $(0.3278,0.3168,0.2793)$ & $0.5772(4)$ \\
$r_{4}$ & $(0.3497,0.2361,0.2372)$ & $0.6255(2)$ & $(0.3484,0.2359,0.2381)$ & $0.6248(2)$ & $(0.3467,0.2357,0.2393)$ & $0.6239(2)$ \\
\hline
\end{tabular}

\section{Conclusions}

In this paper, we give the concept of generalized single-valued neutrosophic deviation measure between two SVNSs, and present an optimal model to generate the attribute weights. If the weight information is completely unknown, the attribute weights can be calculated by using a simple and exact formula. If the weight information is partially available, the information can be easily integrated into the proposed model, where the attribute weights are generated by solving a mathematical programming model and the subjective information of attribute weights converts the constraints. Moreover, the proposed model can be further extended to study the weighting model for the MADM problem with interval valued neutrosophic sets (IVNSs). Şahin and Liu [13] also proposed an approach by maximizing the deviation of attribute values. However, there are the following main advantages of the presented method compared with the existing approach in [13]:

(i) The single-valued neutrosophic deviation measure is general to describe the difference of SVNSs. The selection of the parameter value $p$ makes the computation more flexible than that proposed in [13], which can reflect the decision maker's preference.

(ii) It is not interpreted that the constraint $\sum_{j=1}^{m} \omega_{j}^{2}=1$ in the model proposed by Şahin and Liu [13] from the viewpoint of MADM, where $\omega_{j}$ is the $j$ th weight of the attribute $G_{j}$. Thus, the optimal solution in their model requires being normalized using the formula $\sum_{j=1}^{m} \omega_{j}=1$. However, the attribute weights obtained by the proposed model (7) do not need be normalized because the results satisfy the formula $\sum_{j=1}^{m} \omega_{j}=1$. Therefore, the subjective information about attribute 
weights is easier to combine the objective weight information based on the deviation measure, which can be added directly into model (7) as the constraints.

(iii) The proposed approach can overcome some shortcomings of the method in [13]. In their method, there may only be an attribute weight is 1 and the other attribute weights are neglected. For instance, the attribute weights are $\omega_{3}=1, \omega_{1}=\omega_{2}=\omega_{4}=0$ in Case 3 of the example illustrated.

Author Contributions: W.X. proposed the generalized single-valued neutrosophic deviation measure and the optimization model to determine the attribute weights. J.C. presented the procedure for ranking all alternatives in the MADM problems and gave comparative analysis. Both authors wrote this paper together.

Acknowledgments: The authors would like to thank the editor and anonymous referees for the constructive comments in improving the contents and presentation of this paper. This work is partially supported by the Scientific Research Program Funded by Hubei Provincial Education Department (No. D20182701, B2017168), and the Humanity and Social Science Youth Foundation of the Ministry of Education of China (No. 14YJC630021).

Conflicts of Interest: The authors declare no conflict of interest.

\section{References}

1. Chen, S.J.; Hwang, C.L. Fuzzy Multiple Attribute Decision Making Methods and Applications; Springer: Berlin, Germany, 1992.

2. Ekel, P.Y.; Martini, J.S.C.; Palhares, R.M. Multicriteria analysis in decision-making under information uncertainty. Appl. Math. Comput. 2008, 200, 501-516. [CrossRef]

3. Bellman, R.E.; Zadeh, L.A. Decision-making in fuzzy environment. Manag. Sci. 1970, 17, B141-B164. [CrossRef]

4. Zimmermann, H.J. Fuzzy Set Theory and Its Applications, 3rd ed.; Kluwer Academic: Dordrecht, The Netherlands, 1996.

5. Deng, H.P. Developments in fuzzy multicriteria analysis. Fuzzy Inf. Eng. 2009, 1, 103-109. [CrossRef]

6. Atanassov, K. Intuitionistic fuzzy sets. Fuzzy Sets Syst. 1986, 20, 87-96. [CrossRef]

7. Smarandache, F. A Unifying Field in Logics. Neutrosophy: Neutrosophic Probability, Set and Logic; American Research Press: Rehoboth, DE, USA, 1999.

8. Wang, H.; Smarandache, F.; Zhang, Y.Q.; Sunderraman, R. Single valued neutrosophic sets. Multisp. Multistruct. 2010, 4, 410-413.

9. Broumi, S.; Bakali, A.; Talea, M.; Smarandache, F.; Selvachandran, G. Computing operational matrices in neutrosophic environments: A matlab toolbox. Neutrosophic Sets Syst. 2017, 18, 58-66.

10. Broumi, S.; Bakali, A.; Talea, M.; Smarandache, F.; Uluçay, V.; Sahin, M.; Dey, A.; Dhar, M.; Tan, R.P.; Bahnasse, A.; et al. Neutrosophic sets: An overview. In New Trends in Neutrosophic Theory and Applications; Smarandache, F., Pramanik, S., Eds.; Pons Editions: Brussels, Belgium, 2018; Volume 2, pp. 403-434.

11. Zhang, Z.; Wu, C. A novel method for single-valued neutrosophic multi-criteria decision-making with incomplete weight information. Neutrosophic Sets Syst. 2014, 4, 35-49.

12. Bausys, R.; Zavadskas, E.K.; Kaklauskas, A. Application of neutrosophic set to multicriteria decision-making by COPRAS. J. Econ. Comput. Econ. Cybern. Stud. Res. 2015, 49, 91-106.

13. Şahin, R.; Liu, P. Maximizing deviation method for neutrosophic multiple attribute decision-making with incomplete weight information. Neural Comput. Appl. 2016, 27, 2017-2029. [CrossRef]

14. Biswas, P.; Pramanik, S.; Giri, B.C. Entropy based grey relational analysis method for multi-attribute decision-making under single valued neutrosophic assessments. Neutrosophic Sets Syst. 2014, 2, 102-110.

15. Mondal, K.; Pramanik, S. Neutrosophic decision-making model of school choice. Neutrosophic Sets Syst. 2015, 7, 62-68.

16. Bausys, R.; Zavadskas, E.K. Multicriteria decision-making approach by VIKOR under interval neutrosophic set environment. J. Econ. Comput. Econ. Cybern. Stud. Res. 2015, 49, 33-48.

17. Peng, J.J.; Wang, J.Q.; Zhang, H.Y.; Chen, X.H. An outranking approach for multi-criteria decision-making problems with simplified neutrosophic sets. Appl. Soft Comput. 2014, 25, 336-346. [CrossRef]

18. Ye, J. Multicriteria decision-making method using the correlation coefficient under single-valued neutrosophic environment. Int. J. Gen. Syst. 2013, 42, 386-394. [CrossRef] 
19. Şahin, R.; Liu, P. Correlation coefficient of single-valued neutrosophic hesitant fuzzy sets and its applications in decision-making. Neural Comput. Appl. 2017, 28, 1387-1395. [CrossRef]

20. Aydogdu, A. On similarity and entropy of single valued neutrosophic sets. Gen. Math. Notes 2015, $29,67-74$.

21. Jiang, W.; Shou, Y. A novel single-valued neutrosophic set similarity measure and its application in multicriteria decision-making. Symmetry 2017, 9, 127. [CrossRef]

22. Pramanik, S.; Biswas, P.; Giri, B.C. Hybrid vector similarity measures and their applications to multi-attribute decision-making under neutrosophic environment. Neural Comput. Appl. 2017, 28, 1163-1176. [CrossRef]

23. Xu, X.; Zhang, L.; Wan, Q. A variation coefficient similarity measure and its application in emergency group decision-making. Syst. Eng. Procedia 2012, 5, 119-124. [CrossRef]

24. Ye, J. Improved cosine similarity measures of simplified neutrosophic sets for medical diagnoses. Artif. Intell. Med. 2015, 63, 171-179. [CrossRef] [PubMed]

25. Şahin, R.; Küçük, A. Subsethood measure for single valued neutrosophic sets. J. Intell. Fuzzy Syst. 2015, 29, 525-530. [CrossRef]

26. Huang, H. New distance measure of single-valued neutrosophic sets and its application. Int. J. Intell. Syst. 2016, 31, 1021-1032. [CrossRef]

27. Şahin R. Cross-entropy measure on interval neutrosophic sets and its applications in multicriteria decision-making. Neural Comput. Appl. 2017, 28, 1177-1187. [CrossRef]

28. Liu, P. The aggregation operators based on Archimedean t-conorm and t-norm for the single valued neutrosophic numbers and their application to decision-making. Int. J. Fuzzy Syst. 2016, 18, 849-863. [CrossRef]

29. Şahin, R.; Liu, P. Possibility-induced simplified neutrosophic aggregation operators and their application to multi-criteria group decision-making. J. Exp. Theor. Artif. Intell. 2017, 29, 769-785. [CrossRef]

30. Şahin, R. Normal neutrosophic multiple attribute decision-making based on generalized prioritized aggregation operators. Neural Comput. Appl. 2017, 1-21. [CrossRef]

31. Şahin, R.; Liu, P. Some approaches to multi criteria decision-making based on exponential operations of simplified neutrosophic numbers. J. Intell. Fuzzy Syst. 2017, 32, 2083-2099. [CrossRef]

32. Wang, Y.M.; Luo, Y. Integration of correlations with standard deviations for determining attribute weights in multiple attribute decision-making. Math. Comput. Model. 2010, 51,1-12. [CrossRef]

33. Küçük, G.; Şahin, R. A novel hybrid approach for simplified neutrosophic decision-making with completely unknown weight information. Int. J. Uncertain. Quantif. 2018, 8, 161-173. [CrossRef]

34. Zhang, H.; Ji, P.; Wang, J.; Chen, X.H. An improved weighted correlation coefficient based on integrated weight for interval neutrosophic sets and its application in multi-criteria decision-making problems. Int. J. Comput. Intell. Syst. 2015, 8, 1027-1043. [CrossRef]

35. Biswas, P.; Pramanik, S.; Giri, B.C. A new methodology for neutrosophic multi-attribute decision- making with unknown weight information. Neutrosophic Sets Syst. 2014, 3, 42-52.

36. Ye, J. Improved correlation coefficients of single valued neutrosophic sets and interval neutrosophic sets for multiple attribute decision-making. J. Intell. Fuzzy Syst. 2014, 27, 2453-2462.

37. Peng, J.J.; Wang, J.Q.; Wang, J.; Zhang, H.Y.; Chen, Z.H. Simplified neutrosophic sets and their applications in multi-criteria group decision-making problems. Int. J. Syst. Sci. 2016, 47, 2342-2358. [CrossRef]

38. Şahin, R.; Zhang, H. Induced simplified neutrosophic correlated aggregation operators for multi-criteria group decision-making. J. Exp. Theor. Artif. Intell. 2018, 30, 279-292. [CrossRef]

39. Zeleny, M. Multiple criteria decision-making (MCDM): From paradigm lost to paradigm regained? J. Multi-Criteria Decis. Anal. 2011, 18, 77-89. [CrossRef]

40. Kim, S.H.; Sang, H.C.; Kim, J.K. An interactive procedure for multiple attribute group decision-making with incomplete information: Range-based approach. Eur. J. Oper. Res. 1999, 118, 139-152. [CrossRef]

41. $\mathrm{Xu}, \mathrm{Z}$. An overview of methods for determining OWA weights. Int. J. Intell. Syst. 2005, 20, 843-865. [CrossRef]

42. Izadikhah, M. Using the hamming distance to extend TOPSIS in a fuzzy environment. J. Comput. Appl. Math. 2009, 231, 200-207. [CrossRef]

(C) 2018 by the authors. Licensee MDPI, Basel, Switzerland. This article is an open access article distributed under the terms and conditions of the Creative Commons Attribution (CC BY) license (http://creativecommons.org/licenses/by/4.0/). 